CrossMark \& click for updates

Cite this: Phys. Chem. Chem. Phys., $2015,17,10274$

Received 30th January 2015, Accepted 10th March 2015

DOI: $10.1039 / c 5 c p 00607 d$

www.rsc.org/pccp

\title{
Combining Raman and infrared spectroscopy as a powerful tool for the structural elucidation of cyclodextrin-based polymeric hydrogels
}

\author{
V. Venuti, ${ }^{* a}$ B. Rossi, ${ }^{\text {bc }}$ F. D'Amico, ${ }^{\text {b }}$ A. Mele, ${ }^{\text {de }}$ F. Castiglione, ${ }^{d}$ C. Punta, ${ }^{d}$ \\ L. Melone, ${ }^{d}$ V. Crupi, $^{a}$ D. Majolino, ${ }^{a}$ F. Trotta, ${ }^{f}$ A. Gessini ${ }^{b}$ and C. Masciovecchio ${ }^{b}$
}

A detailed experimental and theoretical vibrational analysis of hydrogels of $\beta$-cyclodextrin nanosponges $(\beta$-CDNS), obtained by polymerization of $\beta$-cyclodextrin $(\beta-C D)$ with the cross-linking agent ethylenediaminetetraacetic acid (EDTA), is reported here. Thorough structural characterization is achieved by exploiting the complementary selection rules of FTIR-ATR and Raman spectroscopies and by supporting the spectral assignments by DFT calculations of the spectral profiles. The combined analysis of the FTIR-ATR spectra of the polymers hydrated with $\mathrm{H}_{2} \mathrm{O}$ and $\mathrm{D}_{2} \mathrm{O}$ allowed us to isolate the $\mathrm{HOH}$ bending of water molecules not involved in symmetrical, tetrahedral environments. The analysis of the $\mathrm{HOH}$ bending mode was carried out as a function of temperature, showing the existence of a supercooled state of the water molecules. The highest level of cooperativity of the hydrogen bond scheme was reached at a value of the $\beta-C D / E D T A$ molar ratio $n=6$. Finally, the connectivity pattern of "uncoupled" water molecules bound to the nanosponge backbone was found to be weakened by increasing $T$. The temperature above which the population of non-tetracoordinated water molecules becomes predominant turned out to be independent of the parameter $n$.

\section{Introduction}

Hydrogels are three-dimensional crosslinked high molecular weight networks formed from a hydrophilic polymer, which have the capability to retain large volumes of water, causing the network to swell in solution. ${ }^{1}$ The network formation can be achieved through covalent-bond cross-linking, as well as by noncovalent interactions such as hydrogen bonding, ionic bonds, van der Waals forces and hydrophobic interactions. ${ }^{2-4}$

During the last few years hydrogels have attracted great interest to biomaterial scientists. ${ }^{5-14}$ Indeed, by a suitable choice of the monomers and polymerization conditions, highly hydrophilic, biocompatible polymers can be obtained, offering interesting loading and release properties. Such capabilities made

${ }^{a}$ Department of Physics and Earth Science, University of Messina, Viale Ferdinando Stagno D'Alcontres 31, 98166 Messina, Italy. E-mail: vvenuti@unime.it

${ }^{b}$ Elettra - Sincrotrone Trieste, Strada Statale $14 \mathrm{~km}$ 163.5, Area Science Park, 34149 Trieste, Italy

${ }^{c}$ Department of Physics, University of Trento, Via Sommarive 14, 38123, Povo, Trento, Italy

${ }^{d}$ Department of Chemistry, Materials and Chemical Engineering "G. Natta", Politecnico di Milano, Piazza L. da Vinci 32, 20133, Milano, Italy

${ }^{e}$ CNR - Istituto di Chimica del Riconoscimento Molecolare, Via L. Mancinelli, 7, 20131 Milano, Italy

${ }^{f}$ Department of Chemistry, University of Torino, Via Pietro Giuria, 10125 Torino, Italy these systems attractive for the delivery of a variety of low molecular weight drugs and bioactive agents such as proteins, peptides, and oligonucleotides, as well as protecting and stabilizing agents for labile molecules. ${ }^{15-18}$ Additionally, the delivery kinetics can be engineered according to the desired drug release schedule by tuning the swelling, the cross-linking density, and the degradation rate. Polymeric hydrogels have been successfully used also as molecular scaffolds for advanced biomedical applications such as cartilage, central nervous system (CNS), and spinal cord injury (SCI) repair therapies. ${ }^{19,20}$ Again, a thixotropic behaviour of the hydrogels is noteworthy, as it allows not only injectability, but also local persistence of the gel once placed in situ. For all these reasons, many types of hydrogels as drug carriers have been widely investigated, and many interesting drug delivery systems based on hydrogels have been proposed..$^{21-23}$ In particular, very recently cyclodextrin (CD)-based hydrogels have gained increasing relevance, since they can combine both the favourable property of CDs to form inclusion complexes and the swelling behaviour of hydrogels. ${ }^{6-9,23,24}$ Cyclodextrins have been physically combined or chemically conjugated with various hydrophilic polymers, such as polysaccharides, various polyvinyl polymers and polyacrylates, in order to achieve desired physicochemical, mechanical, and biopharmaceutical properties. $^{25-28}$

Unfortunately, the synthesis of such conjugates is often complex and requires the use of harsh conditions or toxic chemicals, 
which can compromise the network integrity and application safety. Again, drug transport in these systems is quite complex, due to the various different mechanisms that are contemporarily present. $^{29}$ Finally, it is worth remarking that the analysis of the influence of the chemical composition on the mechanical, physicochemical, and biopharmaceutical properties of these systems requires an in-depth knowledge of their structural properties, as obtained by spectroscopic and mechanical characterization and by swelling investigations.

In this framework, cyclodextrin nanosponges (CDNS), crosslinked polymers prepared by polycondensation between cyclodextrins (CD) and suitable cross-linking agents, have been proved to generate hydrogels with intriguing properties. ${ }^{30-32}$ These systems are, as a matter of fact, super-absorbents for water, the property that makes CDNS hydrogels very interesting for applications of high value and high social impact, such as tissue engineering and regenerative medicine. ${ }^{33-35}$

The structural and dynamic characterization of CDNS hydrogels represents a pivotal prerequisite in view of a rational design of new smart hydrogels with tunable inclusion/release properties. We recently proved that CDNS hydrogels undergo a gel to liquid suspension phase transition by a gradual increase of the hydration level. ${ }^{36-38}$ The results of a deep vibrational dynamics analysis, performed on different regimes by Raman and infrared spectroscopies, evidenced that the macroscopic properties of the gel phase, such as the water holding capacity and the rigidity of the gel network, are closely related to the complex interplay in which physical and covalent interactions combine over different length-scales to yield the formation and stabilization of the hydrogel network. ${ }^{36-40}$ These studies appear to be of particular practical interest because a full understanding of the phenomena of gel-sol transitions observed for CDNS hydrogels is an essential step for the engineering of these systems as biomaterials for tribological uses, for example.

Vibrational analysis of CDNS hydrogels revealed a significant growth of the population of the water molecules with tetrahedral connectivity as the hydration level was increased. The tetrahedral coordination is typical of "bulk" water. This arrangement turned out to be the preferred one with respect to less cooperative motives of water aggregation beyond a characteristic cross-over hydration level $h_{\text {cross }}$. In turn $h_{\text {cross }}$ was found to be dependent (i) on the molar ratio $n$ between cyclodextrin and the cross-linking agent during the synthesis, and (ii) on the type of cyclodextrin used in the polymer synthesis $(\alpha-, \beta$ - or $\gamma$-CD).

Based on these results, we recently explored in detail ${ }^{41}$ the phase behaviour of $\alpha$-, $\beta$-, and $\gamma$-CD nanosponge hydrogels, using the values of $h_{\text {cross }}$ measured via Fourier transform infrared spectroscopy in attenuated total reflection geometry (FTIR-ATR) as a suitable descriptor. It was found that the swelling ability on a macroscopic scale, described by $m=1 / h_{\text {cross }}$, and the elasticity on a mesoscopic scale, described by the Boson Peak frequency $\omega_{\mathrm{BP}}{ }^{42,43}$ undergo the same influence on the molar ratio $n$. We briefly remind here that the so called Boson Peak BP is a peculiar vibrational feature observed in the inelastic light and neutron scattering spectra of disordered systems, ${ }^{44}$ whose origin is still a subject of debate. However, it is known that the position of its maximum moves to higher energies as the stiffness of the material increases. ${ }^{45,46}$

These findings appeared to be useful for the design of the microscopic and macroscopic properties of CDNS by varying the molar ratio $n$ during the synthesis.

In a previous paper, porous morphology and structural heterogeneities in the pore size distribution of $\beta$-CDNSEDTA nanosponges, obtained by polymerization of $\beta$-CD with an activated di-anhydride form of ethylenediaminetetraacetic acid (EDTA), have been obtained using Transmission Electron Microscopy (TEM). ${ }^{47}$ In the present work, the structural elucidation of the hydrated $\beta$-CDNSEDTA network is proposed starting from the analysis of the fundamental normal modes calculated at the Density Functional Theory (DFT) level, and based on the comparison between experimental and calculated IR and Raman spectra. We have chosen to spot this work on nanosponge samples obtained by polymerization of $\beta$-CD with EDTA because this specific formulation of CDNS appears to be particularly promising for the encapsulation and delivery of active agents, as recently demonstrated in the case study of ibuprofen. ${ }^{47}$

As is well known, ${ }^{48}$ these two spectroscopic methods can provide, in several cases, complementary information due to their different selection rules. Symmetric vibrations and ring breathing modes are generally strong in Raman scattering, but forbidden (or, in any case, weaker) in the IR spectra; on the other hand, several asymmetric vibrations along the bonds with a permanent (and strong) dipole moment are more easily detected in IR than in Raman spectroscopy. It is important to remind here that the main parameters, i.e. centre-frequencies and intensities, are generally strongly dependent on the overall chemical and physical properties of the molecule under investigation, well expressed in the so-called "fingerprint" region. A detailed band assignment thus requires massive use of quantum chemical calculations aimed at reproducing both IR/Raman vibrational frequencies and intensities.

Finally, the hydration of our systems by water and deuterated water allowed us to study the different vibration modes of the water molecules - $\mathrm{HOH}$ bending and $\mathrm{O}-\mathrm{H}$ stretching modes - as a function of temperature and $\beta-\mathrm{CD} / \mathrm{EDTA}$ molar ratio $n$. This approach permitted us to separate the vibrational contributions coming from engaged water molecules from those ascribed to the polymeric matrix, thus providing precious information on the hydrogen bond structural organization of confined water.

\section{Results and discussion}

As a first step, a simple model for DFT calculations accounting for the molecular environment of EDTA after the reaction with the $\mathrm{OH}$ groups of cyclodextrins was used. The formation of a methyl di-ester of EDTA was simulated, affording a model of the cross-linker of CDNSEDTA characterized by two ester groups and two free carboxylic groups per EDTA unit. This model was built under the reasonable assumption that no more than two carboxyl groups of EDTA can simultaneously be engaged to form ester linkages with CD molecules (see Scheme 1). 


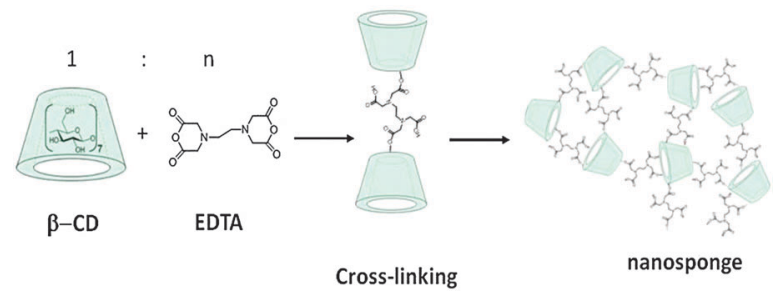

Scheme 1 Scheme of synthesis of $\beta$-CDNS by using activated EDTA as a cross-linking agent; the number $n$ refers to the molar ratio between the reagents.

Fig. 1 reports the simulated and experimental Raman and Infrared spectra in the $1500-1800 \mathrm{~cm}^{-1}$ region for the $\beta$-CDNSEDTA14 nanosponge hydrogel.

As can be clearly seen, in the region of interest $\left(1500-1800 \mathrm{~cm}^{-1}\right)$ Raman and FTIR-ATR spectra appear to be complementary. This was in a way expected, considering that the simulated molecule has an inversion symmetry.

Fig. 2 reproduces a schematic picture of the vibrational modes obtained for the simulated bridging molecule.

Two characteristic types of vibration modes can be observed in this frequency range: asymmetric (IR active/Raman inactive) and symmetric (IR inactive/Raman active) stretchings of the $\mathrm{C}=\mathrm{O}$ groups (carboxylic and ester), and asymmetric (IR active/ Raman inactive) and symmetric (IR inactive/Raman active) bendings of the $\mathrm{CH}_{2}$ groups as reported in the figure.

The comparison of the experimental Raman and FTIR-ATR spectra obtained for the $\beta$-CDNSEDTA14 hydrogel (Fig. 1, the panel at the bottom) can be conveniently done by using the hydrogels prepared in $\mathrm{D}_{2} \mathrm{O}$ instead of $\mathrm{H}_{2} \mathrm{O}$ in order to eliminate the interfering contribution due to the bending mode of water molecules, falling at $\sim 1640 \mathrm{~cm}^{-1}$, which will be discussed in depth in the following. At the moment, we are focusing on the vibrational modes of the nanosponge network when hydrated.

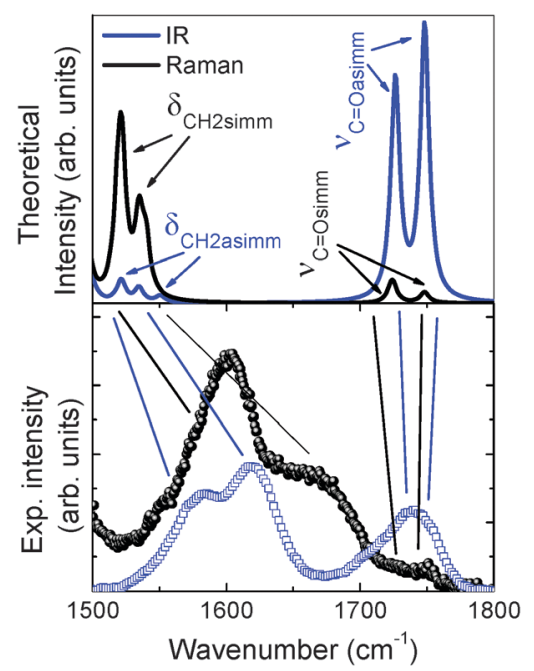

Fig. 1 (top) Theoretical Raman (black line) and Infrared (blue line) intensities obtained for the simulated bridging molecule. (bottom) Experimental polarized Raman intensity (black circles) and FTIR-ATR absorbance (open squares) obtained for the $\beta$-CDNSEDTA14 nanosponge hydrogel.
As can be observed, the differences between the Raman and FTIR-ATR spectra recall those predicted by the DFT calculations, thus providing a cross-check for the proposed model and a support for the band assignment.

Fig. 3 shows the superimposition of Raman and FTIR-ATR spectra of hydrogels obtained by hydration, in water, of different $\beta$-CDNSEDTA1 $n(n=4,6,8,10)$ samples. In order to make the comparison easier, both Raman and IR spectra have been normalized to the maximum of the symmetric and asymmetric CCO stretching modes, respectively.

IR spectra are particularly sensitive to the presence of water, which in the spectral region of interest exhibit a contribution at $\sim 1640 \mathrm{~cm}^{-1}$ ascribed to the $\mathrm{HOH}$ bending mode. As wellestablished, ${ }^{49-51}$ the bending band is commonly assigned to the water molecules not involved in a symmetric tetrahedral network. (Incidentally, it is worth recalling that such a band, in liquid water, undergoes reduced intensity and increased frequency upon cooling.) Since this mode is usually overlapped to the vibrational bands of the polymer, suitable spectral subtraction of the vibrational profiles of the hydrogels prepared with $\mathrm{D}_{2} \mathrm{O}$ instead of $\mathrm{H}_{2} \mathrm{O}$ was accomplished in order to isolate the vibrational modes of the water molecules.

Fig. 4 shows, as an example, the FTIR-ATR spectra of the hydrogels prepared by hydration of $\beta$-CDNSEDTA1 8 in $\mathrm{H}_{2} \mathrm{O}$ and $\mathrm{D}_{2} \mathrm{O}$, together with the corresponding difference spectrum, where the profile in deuterated water is subtracted from the corresponding profile in $\mathrm{H}_{2} \mathrm{O}$. The spectra in $\mathrm{H}_{2} \mathrm{O}$ and $\mathrm{D}_{2} \mathrm{O}$ have been preliminarily normalized to the intensity of the band at about $1030 \mathrm{~cm}^{-1}$, assumed as a reliable internal standard. ${ }^{39,42}$ Indeed, the latter vibrational mode is related to stretching vibrations of $\mathrm{C}-\mathrm{O}$ groups of cyclodextrin molecules, which are not expected to be affected by the hydration of the nanosponge or by a variation in temperature.

The profiles of $\mathrm{D}_{2} \mathrm{O}$ hydrogels trace, within the experimental error, the corresponding spectra in $\mathrm{H}_{2} \mathrm{O}$ except for the component of the $\mathrm{HOH}$ bending mode, which exhibit a characteristic Gaussian-like form. This finding confirms the reliability of the data handling followed for the subtraction of the spectra.

In the case of bulk water, as widely reported and discussed in the literature ${ }^{50,52}$ by different authors, the experimental IR spectra acquired in the temperature range between 264 and $320 \mathrm{~K}^{50,53}$ show that, unlike the $\mathrm{OH}$ stretching band, the $\mathrm{HOH}$ bending mode decreases in intensity upon cooling and almost vanishes at crystallization. Moreover, as the temperature is lowered, the position of its maximum shifts towards a higher wavenumber. ${ }^{50}$ This behaviour suggests the interpretation that the bending band mostly reflects the population of water molecules that do not lie in a symmetric tetrahedral environment, ${ }^{50}$ giving an indication that the $\mathrm{HOH}$ bending mode is hardly sensitive to the different levels of connectivity of HB patterns developed by $\mathrm{H}_{2} \mathrm{O}$. It is therefore interesting to examine the evolution of the centre-frequency and intensity of this band for our samples as a function of $T$. A qualitative trend can be detected by the inspection of the raw FTIR-ATR profiles reported in the inset of Fig. 4 for the $\beta$-CDNSEDTA110 hydrogel in the whole range of $T$ explored as an example. Two main points can 

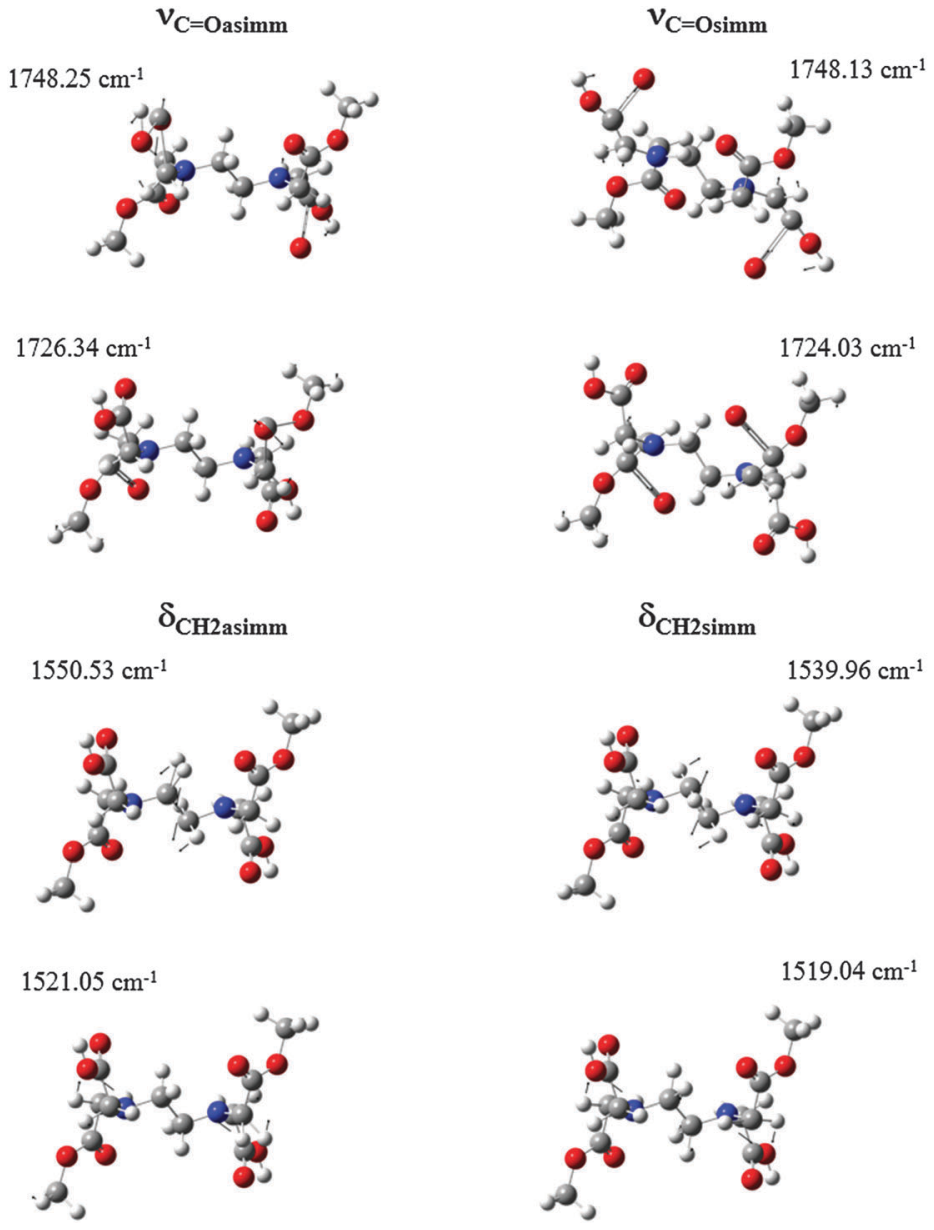

Fig. 2 Schematic picture of the vibrational modes obtained for simulated bridging molecules in the spectral window $1500-1800 \mathrm{~cm}^{-1}$.

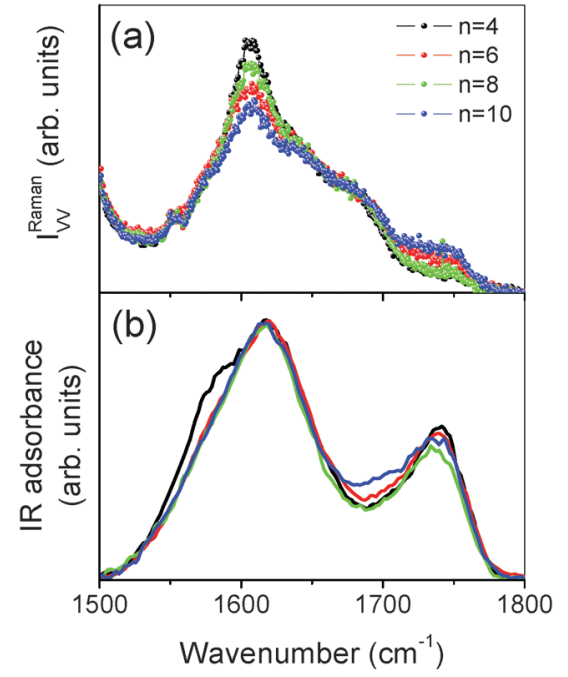

Fig. 3 Polarized Raman intensity (a) and IR adsorbance (b) obtained for $\beta$-CDNSEDTA1 $n$ nanosponge hydrogels $(n=4,6,8$ and 10$)$.

be underlined: (i) the existence, at $T=250 \mathrm{~K}$, of a contribution different from the nearly flattened profile typically exhibited by polycrystalline ice, and (ii) an increasing intensity together with a slight shift to a lower wavenumber upon increasing temperature.

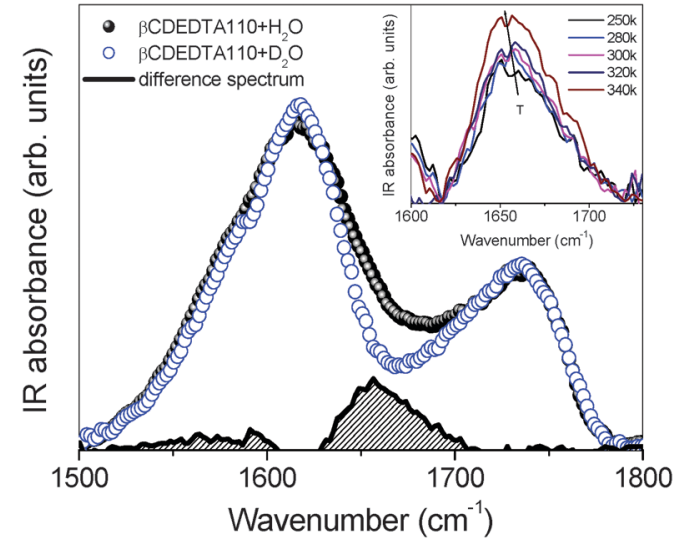

Fig. 4 FTIR-ATR spectra in the wavenumber range $1500-1800 \mathrm{~cm}^{-1}$ of the $\beta$-CDNSEDTA110 hydrogel obtained by hydration of corresponding nanosponges in $\mathrm{H}_{2} \mathrm{O}$ (black symbols) and $\mathrm{D}_{2} \mathrm{O}$ (open circles). The line represents the difference spectrum (see text for details). In the inset, the evolution vs. $T$ of the $\mathrm{HOH}$ bending mode for the same sample, as obtained by raw FTIR-ATR profiles, is reported.

In order to go deeper inside the temperature effects on the $\mathrm{HOH}$ bending mode, a quantitative analysis was performed, isolating the bending contribution from the spectra. First of all, we fitted the FTIR-ATR spectrum of the hydrogels prepared in $\mathrm{D}_{2} \mathrm{O}$ in 
the 1500-1800 $\mathrm{cm}^{-1}$ wavenumber range for each nanosponge by using Voigt functions. In this way, the bands ascribed to the vibration modes of a polymeric matrix have been detected without the interfering contribution of the bending mode that, in this case, shifts to lower frequencies. The so obtained number of bands and centre-frequencies have been then fixed (thus reducing the number of free fitting parameters) and used as a starting point for the fit performed, in the same range, for the corresponding hydrogel prepared in $\mathrm{H}_{2} \mathrm{O}$. We also added the band assigned to the $\mathrm{HOH}$ bending mode of water molecules. It is worth remarking that the adopted fitting procedure is well documented in the literature and it has been successfully applied to the analysis of the IR active molecular modes of nanosponges in dry ${ }^{42}$ and hydrated states. ${ }^{36,38-40,54}$ The choice of a Voigt function (as specified in the Experimental section) also for reproducing the $\mathrm{HOH}$ bending mode of water, although arbitrary, is quite standard to obtain in a more quantitative way the intensity and wavenumber position of the bending mode. An example of the fitting strategy used is reported in Fig. 5 for $\beta$-CDNSEDTA16 nanosponges at $T=260 \mathrm{~K}$.

The temperature evolution of the $\mathrm{HOH}$ bending contribution of $\mathrm{H}_{2} \mathrm{O}$ molecules, in the $T$-range extending from $T=250 \mathrm{~K}$ to $T=340 \mathrm{~K}$, is reported in Fig. 6(a) and (b) for $\beta$-CDNSEDTA14 and $\beta$-CDNSEDTA18 hydrogels, respectively, as an example. The same behaviour has been revealed for all the investigated systems.

The overall trend of this band is the same as that observed in the raw IR profiles (see the inset of Fig. 4), thus confirming that the fitting procedure described does not affect the results.

The destructuring effect of increasing temperature on the connectivity pattern of the hydrogen bond network, as commonly revealed in many hydrogen-bonded systems, ${ }^{55}$ is clearly highlighted by the growth of the number of water molecules involved in loosely tight transient environments, as indicated by the lowenergy shift of the band maximum and, at the same time, the progressive increase of its intensity. Again, it is worth remarking

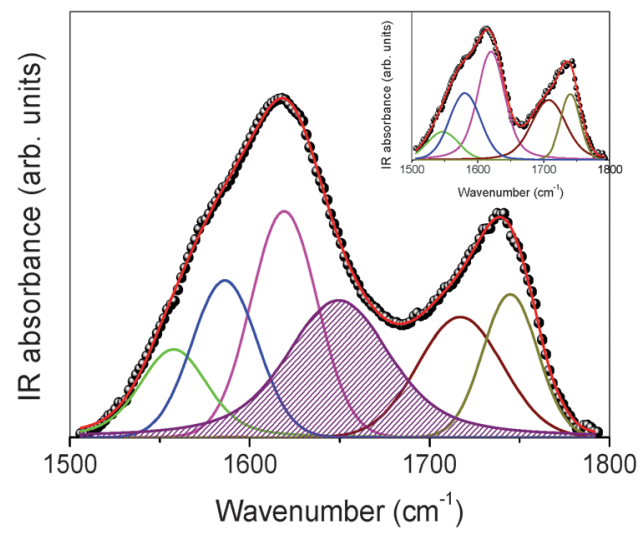

Fig. 5 Fitting results (best-fit: red line, individual components: coloured lines) for the FTIR-ATR spectrum (closed circles), in the $1500-1800 \mathrm{~cm}^{-1}$ range, at $T=260 \mathrm{~K}$, of $\beta$-CDNSEDTA16 hydrogels obtained by hydration of corresponding nanosponges in $\mathrm{H}_{2} \mathrm{O}$. The filled area indicates the $\mathrm{HOH}$ bending contribution of water. In the inset, the case corresponding to $\beta$-CDNSEDTA16 hydrogels prepared in $\mathrm{D}_{2} \mathrm{O}$ is reported (see text for details).

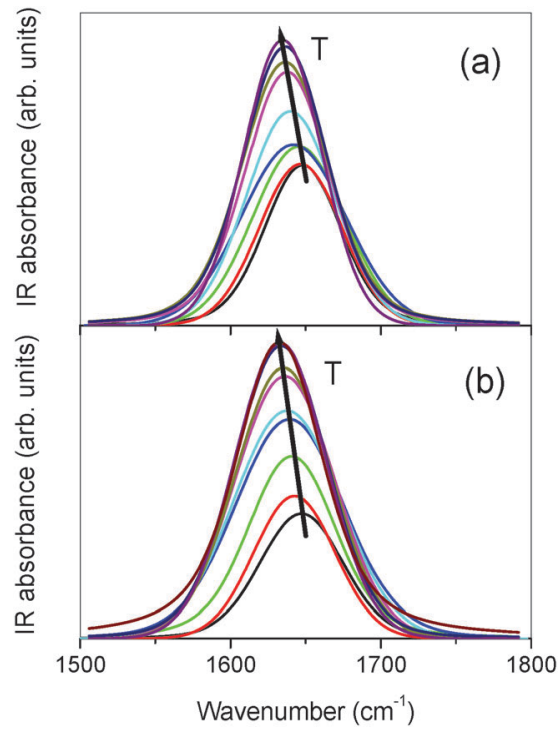

Fig. 6 Temperature evolution of the $\mathrm{HOH}$ bending band of water molecules for $\beta$-CDNSEDTA14 (a) and $\beta$-CDNSEDTA18 (b) hydrogels.

the presence of a non-null intensity of the bending contribution even at the lowest analysed temperature, below the crystallization temperature of bulk water. This occurrence furnishes evidence of a supercooled behaviour of confined water, i.e. transient $\mathrm{H}$-bond arrangements with a mean coordination number less than four are still present, in full agreement with what was already revealed for $\beta$-CDNSPMA nanosponges ${ }^{40}$ and for a variety of hydrated nanoporous systems. ${ }^{56-59}$

In Fig. 7 the temperature dependence of the centre-frequencies of the $\mathrm{HOH}$ bending band is reported for all the investigated systems.

Fig. 7 indicates that the maximum value of the position of the $\mathrm{HOH}$ bending band, corresponding to the maximum number of water molecules involved in symmetrical, bulk-like, tetrahedral patterns, is always reached at $n=6$, irrespective of $T$. This means that, at least in the explored temperature range,

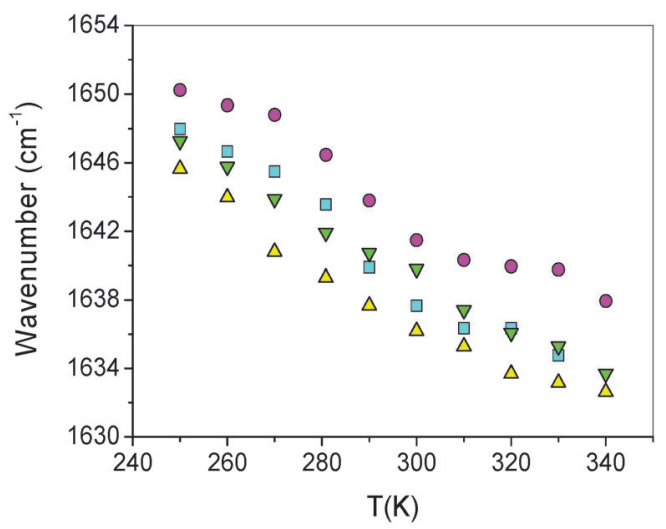

Fig. 7 Temperature evolution of the position of the maximum of the $\mathrm{HOH}$ bending band of water molecules for $\beta$-CDNSEDTA14 (squares), $\beta$-CDNSEDTA16 (circles), $\beta$-CDNSEDTA18 (up triangles), and $\beta$-CDNSEDTA110 (down triangles) hydrogels. 
the hydrogen bond network exhibits the highest level of cooperativity in $\beta$-CDNSEDTA16 nanosponges. This result appears to be in full agreement with the analysis of the $\mathrm{O}-\mathrm{H}$ stretching profile done, at room temperature, on $\alpha$-, $\beta$ - and $\gamma$-CDNSEDTA nanosponge hydrogels prepared in $\mathrm{H}_{2} \mathrm{O}$ at different levels of hydrations. ${ }^{33,38}$ It confirms, once again, that the cross-linker/ monomer molar ratio $n$ is a key parameter for the modulation of the chemical/physical properties of ester-based CDNS.

The spectral window $3000-3800 \mathrm{~cm}^{-1}$ in the FTIR-ATR spectra of the hydrogels prepared in $\mathrm{D}_{2} \mathrm{O}$ deserves some comments.

As suggested by the other authors in the case of aqueous solutions of proteins, ${ }^{59}$ the use of $\mathrm{D}_{2} \mathrm{O}$ as a solvent allows us to selectively probe the $\nu_{\mathrm{OH}}$ modes of water molecules resulting from isotopic exchanges between the superficial $\mathrm{H}$ atoms of CDNS and the solvent $\mathrm{D}_{2} \mathrm{O}$ placed closely around the polymer surface, namely DHO water molecules. These water molecules contribute to the IR spectrum in the $\mathrm{OH}$ stretching region between 2800 and $3800 \mathrm{~cm}^{-1}$, which appears to be significantly different from that observed in $\mathrm{H}_{2} \mathrm{O}$, as pointed out in the inset of Fig. 8 in the case of $\beta$-CDNSEDTA110 at $T=290 \mathrm{~K}$ as an example (no interference from the $\mathrm{O}-\mathrm{D}$ stretching mode of $\mathrm{D}_{2} \mathrm{O}$ molecules is present, as such a band is in the $2000-2800 \mathrm{~cm}^{-1}$ range). This occurrence, in agreement with previous studies, ${ }^{60-62}$ is consistent with the hypothesis that the DHO spectrum reflects a population of water molecules, which more strongly interact with the chemical groups of CDNS within the nano-cavities of the polymer in hydrogels. Consequently, the DHO spectrum can be considered to be free of intermolecular coupling vibrations and is called the uncoupled stretching region. ${ }^{59}$

The evolution of the vibrational profile in the spectral region mentioned above, as a function of temperature, is plotted in Fig. 8 for $\beta$-CDNSEDTA110 nanosponges as an example. In the figure, the band observed between 2800 and $3000 \mathrm{~cm}^{-1}$ is ascribed to the $\mathrm{C}-\mathrm{H}$ stretching mode, to which the $\mathrm{O}-\mathrm{H}$ mode is convoluted.

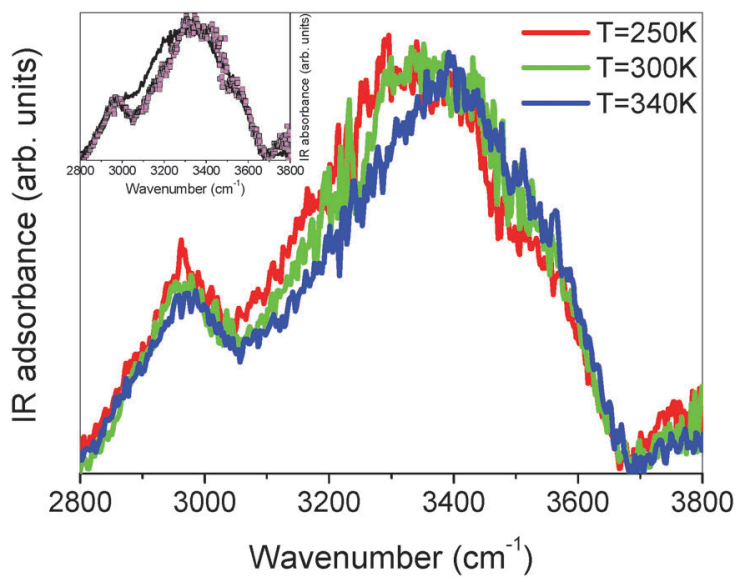

Fig. 8 Temperature evolution of the $\mathrm{O}-\mathrm{H}$ stretching band of $\beta$-CDNSEDTA110 hydrogels prepared in $\mathrm{D}_{2} \mathrm{O}$. In the inset, the $\mathrm{O}-\mathrm{H}$ stretching band, measured at $T=290 \mathrm{~K}$, of $\beta$-CDNSEDTA110 hydrogels prepared in $\mathrm{H}_{2} \mathrm{O}$ (continuous line) and in $\mathrm{D}_{2} \mathrm{O}$ (squares).
The inset of Fig. 8 clearly shows that the width of the $\mathrm{O}-\mathrm{H}$ band of the hydrogel in $\mathrm{D}_{2} \mathrm{O}$ is smaller than the corresponding width for the system in $\mathrm{H}_{2} \mathrm{O}$, indicating a moderately strong $\mathrm{H}$ bond network for these samples.

The spectra in Fig. 8 display, by increasing $T$, an overall shift of the band towards higher wavenumbers, together with a progressive enhancement of the high-frequency contribution at the expense of the low-frequency one. This finding suggests a decrease in the co-operativity in the H-bond scheme, so confirming the destructuring effect of temperature already put into evidence by the analysis of the $\mathrm{HOH}$ bending mode. As widely reported in the literature, ${ }^{60,63-65}$ a detailed picture of the connectivity pattern of water molecules inside the polymeric matrix can be obtained by the deconvolution of the $\mathrm{O}-\mathrm{H}$ stretching band by means of a curve-fitting procedure. Based on a well-established model, ${ }^{66,67}$ already successfully applied to the analysis of the $\mathrm{OH}$ stretching band in Raman and IR spectra of CDNS hydrogels, ${ }^{36-40}$ the spectral changes observed in the $\mathrm{O}-\mathrm{H}$ stretching profiles are analysed in terms of several classes of $\mathrm{O}-\mathrm{H}$ oscillators, involved in transient $\mathrm{H}$-bonded and non $\mathrm{H}$-bonded arrangements, of water more closely confined in the pores of CDNS polymers.

First of all, because of the partial overlapping of $\mathrm{O}-\mathrm{H}$ and $\mathrm{C}-\mathrm{H}$ stretching bands, it was necessary to fit the whole spectrum in order to separate the $\mathrm{O}-\mathrm{H}$ stretching region. Voigt bands were used as fitting functions, and the contributions due to symmetric and asymmetric methyl stretches needed to be subtracted from the total fits. More detailed fits were subsequently performed just in the $\mathrm{O}-\mathrm{H}$ stretching region. In particular, four different contributions have been recognized, as evidenced in Fig. 9, as examples of the best curve-fitting results for $\beta$-CDNSEDTA14 at $T=310 \mathrm{~K}$.

The two sub-bands at the lowest wavenumbers, $\omega_{1}$ and $\omega_{2}$, are, respectively, ascribed to symmetric and asymmetric $\mathrm{O}-\mathrm{H}$ stretching modes of water molecules in tetrahedral connectivity exhibiting strong $\mathrm{H}$-bonds on both the hydrogen atoms. The third sub-band $\omega_{3}$ is assigned to the non-in-phase $\mathrm{O}-\mathrm{H}$ mode of tetrahedral environments linked by the "bifurcated" H-bond. Finally, the spectral component $\omega_{4}$ represents the O-H mode of

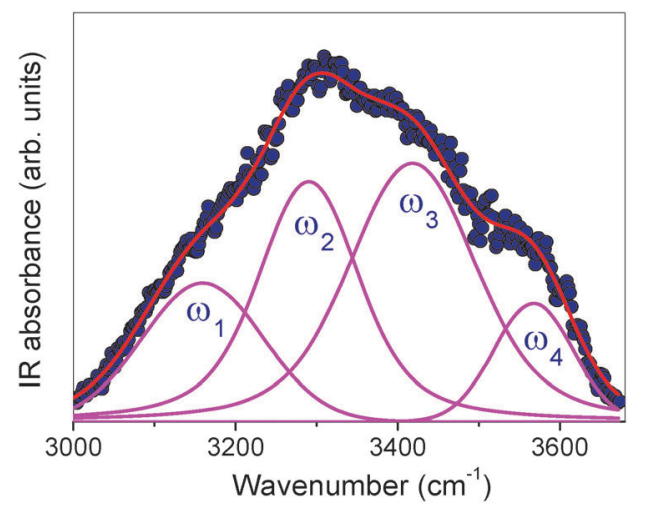

Fig. 9 Fitting results (best-fit: red line, individual components: pink lines) for the FTIR-ATR spectrum (closed circles), in the $3000-3700 \mathrm{~cm}^{-1}$ range, at $T=310 \mathrm{~K}$, of $\beta$-CDNSEDTA14 hydrogels obtained by hydration of corresponding nanosponges in $\mathrm{D}_{2} \mathrm{O}$. 

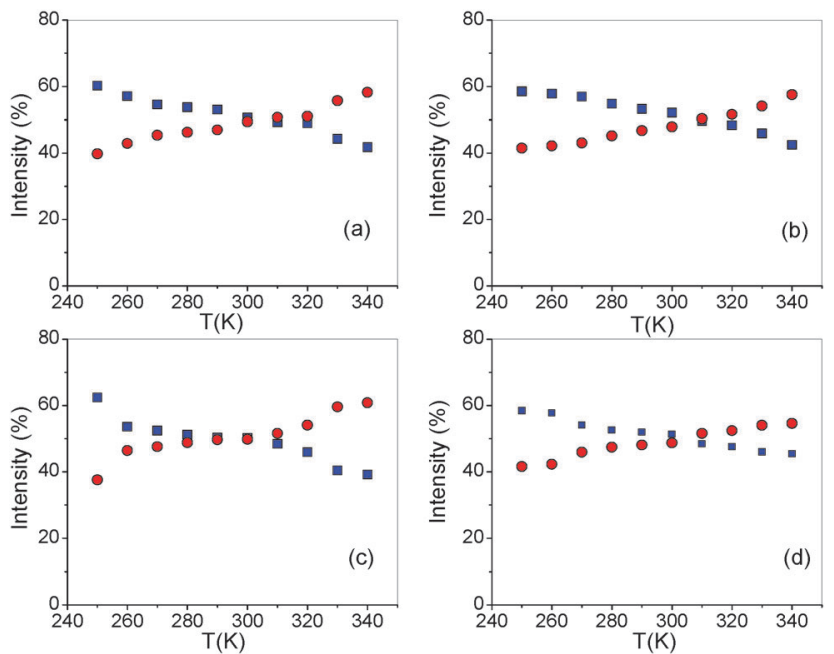

Fig. 10 Evolution, as a function of $T$, of the percentage intensities $I_{1}+I_{2}$ (blue squares) and $I_{3}+I_{4}$ (red circles) of the spectral contributions to the $\mathrm{OH}$ stretching band for $\beta$-CDEDTA14 (a), $\beta$-CDEDTA16 (b), $\beta$-CDEDTA18 (c), and $\beta$-CDEDTA110 (d) hydrogels obtained by hydration of corresponding nanosponges in $\mathrm{D}_{2} \mathrm{O}$

those $\mathrm{H}_{2} \mathrm{O}$ molecules for which the $\mathrm{H}$-bond network is, totally or at least partially, destroyed. Labelled as $I_{i}(i=1,2,3,4)$ the estimated percentage intensities of each contribution, the sum $I_{1}+I_{2}$ will be representative of the population of tetra-coordinated water molecules, whereas $I_{3}+I_{4}$ will account for the population of water molecules involved in H-bond arrangements with a lower coordination degree. Their evolution versus $T$ is reported in Fig. 10 for all the investigated hydrogels in $\mathrm{D}_{2} \mathrm{O}$.

The enhancement of temperature is found to increase, at the expense of the highly connective species, low-coordinated structures, which will become favoured at a characteristic cross-over point observed at about $T=300-310 \mathrm{~K}$ for all the $\beta$-CDEDTA1 $n$ $(n=4,6,8,10)$ hydrogels in $\mathrm{D}_{2} \mathrm{O}$.

\section{Experimental}

\section{Materials}

In order to obtain $\beta$-CDNSEDTA1 $n$ nanosponges, anhydrous $\beta$ CD was dissolved at room temperature in anhydrous DMSO containing anhydrous $\mathrm{Et}_{3} \mathrm{~N}$. Then, the cross-linking agent ethylenediaminetetraacetic acid (EDTA) dianhydride was added at molecular ratios of $1: n$ (with $n=4,6,8,10$ ) under intense magnetic stirring. Polymerization was complete in a few minutes by obtaining a solid that was broken up with a spatula and washed with acetone in a Soxhlet apparatus for $24 \mathrm{~h}$. The pale yellow solid was finally dried under vacuum.

The corresponding hydrogels of nanosponges were prepared by adding to the dry samples of $\beta$-CDNSEDTA1 $n(n=4,6,8,10)$ the suitable amount of double-distilled water (Sigma) or heavy water (Sigma) in order to obtain the desired level of hydration $h$, defined, respectively, as the weight ratio of $\mathrm{H}_{2} \mathrm{O} / \beta$-CDNSEDTA1n or $\mathrm{D}_{2} \mathrm{O} / \beta$-CDNSEDTA $1 n$.

All the gel samples were freshly prepared and used for UV Raman and FTIR-ATR measurements.

\section{UV Raman spectroscopy measurements}

UV Raman scattering measurements were carried out at the BL10.2-IUVS beamline at the Elettra Synchrotron laboratory in Trieste. ${ }^{68}$ Data have been collected at room temperature on $\beta$-CDNSEDTA1 $n(n=4,6,8,10)$ hydrogels prepared by hydration in $\mathrm{H}_{2} \mathrm{O}$ and $\mathrm{D}_{2} \mathrm{O}$ of the corresponding dry samples of CDNS. The analysed hydration level was $h=4$. The spectra were excited at $266 \mathrm{~nm}$ and collected in a back-scattered geometry using a triple stage spectrometer (Trivista, Princeton Instruments). The experimental resolution was set to $1.0 \mathrm{~cm}^{-1}$ in order to ensure enough resolving power and count-rate. To minimize potential photodecomposition of the gels resulting from UV exposure, the sample cell was subjected to slow continuous spinning during the measurements in order to vary the illuminated sample volume through the radiation beam. A complete detailed description of the experimental set-up can be found elsewhere. ${ }^{69}$ By using polarizers and wave plates, we have collected the light inelastically diffused from the sample polarized parallel with respect to the incident radiation.

\section{FTIR-ATR spectroscopy measurements}

FTIR-ATR measurements were performed in the temperature range extending from $T=250 \mathrm{~K}$ to $T=340 \mathrm{~K}$ on $\beta$-CDNSEDTA1 $n$ $(n=4,6,8,10)$ hydrogels prepared by hydration in $\mathrm{H}_{2} \mathrm{O}$ and $\mathrm{D}_{2} \mathrm{O}$ of the corresponding dry samples of CDNS. The analysed hydration level was $h=0.4$. We remark that the hydration level used for IR measurements is lower with respect to that adopted for Raman experiments because the vibrational modes of water appear to be particularly intense in the IR spectra compared to the Raman profile, even at low hydration levels. At the same time, low values of hydration allow us to better analyse in the IR spectra of hydrogels also the vibrational bands associated with the specific vibrating groups of the polymer. The spectra were collected on a Bomem DA8 Fourier transform spectrometer, operating with a Globar source, in combination with a $\mathrm{KBr}$ beamsplitter, a DTGS/KBr detector. Spectra were collected in the $400-4000 \mathrm{~cm}^{-1}$ wavenumber range. The samples were contained in the Golden Gate diamond ATR system, just based on the ATR technique. ${ }^{70}$

An ATR setup exhibits various advantages with respect to an ordinary absorption setup. It is non-destructive, it requires only micrograms of the sample, and it is at the origin of spectra displaying a very good signal-to-noise ratio, being in particular easy to avoid saturation of bands. In addition, a chemical analysis can be performed directly on ATR spectra, avoiding implementation of elaborated calculations of optical constants. The spectra were recorded in dry atmosphere, in order to avoid dirty contributions, with a resolution of $4 \mathrm{~cm}^{-1}$, automatically adding 100 repetitive scans in order to obtain a good signal-tonoise ratio and high reproducibility. All the IR spectra were normalized for taking into account the effective number of absorbers. No mathematical correction (e.g., smoothing) was done, and spectroscopic manipulation such as baseline adjustment and normalization were performed using the Spectracalc software package GRAMS (Galactic Industries, Salem, NH, USA). For the $1500-1800 \mathrm{~cm}^{-1}$ region, second derivative computations 
(not reported here) have been used for evaluating the wavenumbers of the maxima of the different sub-bands. Multiple curve fitting into Voigt profiles were then applied to the experimental profiles based on these wavenumber values, by using the routine provided in the PeakFit 4.0 software package. The statistical parameters defined in the software manual were used as a guide to the best-fit and allowed to vary upon iteration until the converging solution is reached. The best-fit is characterized by $r^{2} \approx 0.9999$ for all the investigated systems.

\section{Computational details}

Molecular models were built by using the PCMODEL 8.0 package (Serena Software, Bloomington, IN, USA) and allowed to fully relax in the MMX force field. The MMX energy minimized geometry was in turn used as a starting structure for $a b$ initio quantum chemical computations carried out using the GAUSSIAN 03 program suite using unrestricted Density Functional Theory (DFT). The nonlocal B3LYP functional hybrid method was employed. The standard $6-31 \mathrm{G}$ basis set was used for the geometry optimization and vibrational energy analysis.

\section{Conclusions}

We present here a combined FTIR-ATR and Raman vibrational analysis of $\beta$-cyclodextrin nanosponges cross-linked by ethylenediaminetetraacetic acid ( $\beta$-CDNSEDTA) in their hydrogel state and at different $\beta$-CD/EDTA molar ratios $n$. The assignment of the vibrational bands was achieved by a suitable simulation of the vibrational profile in a model compound at the DFT level. The hydration of the nanosponges in $\mathrm{H}_{2} \mathrm{O}$ and $\mathrm{D}_{2} \mathrm{O}$ was carried out in order to separate the contributions of the engaged water molecules from the vibrational modes assigned to specific chemical groups of the polymer matrix involved in the physical interactions with water. The approach proposed here has three main advantages: (i) the joint analysis of Raman and IR spectra of CDNS allows us to achieve thorough characterization, at the molecular level, of the structure of the polymer by exploiting the complementary selection rules of FTIR-ATR and Raman spectroscopies, (ii) the swelling of the polymer in both $\mathrm{H}_{2} \mathrm{O}$ and $\mathrm{D}_{2} \mathrm{O}$ allowed for a complete structural elucidation of the polymeric network in its hydrated state, and (iii) the $\mathrm{HOH}$ bending FTIRATR vibration of water molecules entrapped in the polymeric matrix could be monitored as a function of $T$, thus providing information on the evolution of the hydrogen bond architecture. A destructuring effect of the thermal motion on the hydrogen bond connectivity was uncovered by the changes observed in wavenumber and intensity of the bending band. The data showed the presence of $\mathrm{H}_{2} \mathrm{O}$ molecules not arranged in tetrahedral coordination even below the water melting point, thus providing evidence of a supercooled state of water in the examined systems. The tetrahedral arrangement of water molecules becomes prevalent at $n=6$, in agreement with the previous results on similar systems, confirming the key role played by the parameter $n$ as obtained by the synthetic process.
Finally, the investigation of the evolution of the FTIR-ATR "uncoupled" $\mathrm{O}-\mathrm{H}$ stretching vibration at variable $T$ and $n$ allowed us to achieve quantitative information, by means of a best-fit procedure, of the hydrogen bond organization of water molecules early bound to the nanosponge network. A characteristic crossover temperature has been observed, above which the population of not bulk-like $\mathrm{H}_{2} \mathrm{O}$ molecules, namely water molecules with a low level of cooperativity, becomes favoured with respect to that of bulk-like, tetracoordinated water. The cross-over temperature, in turn, was found not to be affected by the molar ratio $n$.

\section{Acknowledgements}

The authors are grateful to Dr Marco Paolantoni (University of Perugia, Italy) for the very fruitful discussions. The authors gratefully acknowledge PRIN 2010-2011 NANOMED prot. 2010 FPTBSH and PRIN 2010-2011 PROxy prot. 2010PFLRJR_005 for financial support.

\section{Notes and references}

1 B. V. Slaughter, S. S. Khurshid, O. Z. Fisher, A. Khademhosseini and N. A. Peppas, Adv. Mater., 2009, 21, 3307.

2 W. E. Hennik and C. F. van Nostrum, Adv. Drug Delivery Rev., 2002, 54, 13.

3 L. Yu and J. D. Ding, Chem. Soc. Rev., 2008, 37, 1473.

4 M. Ma, Y. Kuang, Y. Gao, Y. Zhang, P. Gao and B. Xu, J. Am. Chem. Soc., 2010, 132, 2719.

5 J. Rault, A. Lucas, R. Neffati and M. M. Pradas, Macromolecules, 1997, 30, 7866.

6 Y. Y. Liu and X. D. Fan, Polymer, 2002, 43, 4997.

7 F. Quaglia, G. Varricchio, A. Miro, M. I. L. Rotonda, D. Larobina and G. Mensitieri, J. Controlled Release, 2001, 71, 329.

8 J. Li, X. Ni and K. W. Leong, J. Biomed. Mater. Res., Part A, 2003, 65, 196.

9 Y. Y. Liu, X. D. Fan, H. Hu and Z. H. Tang, Macromol. Biosci., 2004, 4, 729.

10 A. S. Hoffman, Adv. Drug Delivery Rev., 2002, 43, 3.

11 N. A. Peppas, P. Bures, W. Leonbandung and H. Ichikawa, Eur. J. Pharm. Biopharm., 2000, 50, 27.

12 J. Nanda, A. Biswas and A. Banerjee, Soft Matter, 2013, 9, 4198. 13 J. Nanda and A. Banerjee, Soft Matter, 2012, 8, 3380.

14 A. Baral, S. Roy, A. Dehsorkhi, I. W. Hamley, S. Mohapatra, S. Ghosh and A. Banerjee, Langmuir, 2014, 30, 929.

15 H. Park and K. Park, Pharm. Res., 1996, 13, 1770.

16 K. R. Kamath and K. Park, Adv. Drug Delivery Rev., 1993, 11, 59.

17 J. L. Cleland, A. Daugherty and R. Mrsny, Curr. Opin. Biotechnol., 2001, 12, 212.

18 D. J. A. Crommelin, G. Storm, W. Jiskoot, R. Stenekes, E. Mastrobattista and W. E. Hennink, J. Controlled Release, 2003, 87, 81 .

19 J. Elisseeff, W. McIntosh, K. Anseth, S. Riley, P. Ragan and R. Langer, J. Biomed. Mater. Res., 2000, 51, 164. 
20 F. Rossi, G. Perale and M. Masi, Chem. Pap., 2010, 64, 573.

21 A. Kikuchi and T. Okano, Adv. Drug Delivery Rev., 2002, $54,53$.

22 X. Z. Zhang, D. Q. Wu and C. C. Chu, Biomaterials, 2004, 25, 3793.

23 K. Sreenivasan, J. Appl. Polym. Sci., 1997, 65, 1829.

24 Y. Y. Liu, X. D. Fan, T. Kang and L. Sun, Macromol. Rapid Commun., 2004, 25, 1912.

25 J. Watanabe, T. Ooya, K. H. Nitta, K. D. Park, Y. H. Kim and N. Yui, Biomaterials, 2002, 23, 4041.

26 F. Zeng-Guo and Z. Sanping, Polymer, 2003, 44, 5177.

27 W. Pluemsab, N. Sakairi and T. Furuike, Polymer, 2005, 46, 9778.

28 U. Siemoneit, C. Schmitt, C. Alvarez-Lorenzo, A. Luzardo, F. Otero-Espinar, A. Concheiro and J. Blanco-Méndez, Int. J. Pharm., 2006, 312, 66.

29 G. Perale, F. Rossi, M. Santoro, P. Marchetti, A. Mele, F. Castiglione, E. Raffa and M. Masi, J. Biomed. Nanotechnol., 2011, 7, 1.

30 A. Mele, F. Castiglione, F. Ganazzoli, G. Raffaini, F. Trotta, B. Rossi and A. Fontana, J. Inclusion Phenom. Macrocyclic Chem., 2011, 69(3-4), 403.

31 W. Liang, C. Yang, M. Nishijima, G. Fukuhara, T. Mori, A. Mele, F. Castiglione, F. Caldera, F. Trotta and Y. Inoue, Beilstein J. Org. Chem., 2012, 8, 1305.

32 W. Liang, C. Yang, D. Zhou, H. Haneoka, M. Nishijima, G. Fukuhara, T. Mori, F. Castiglione, A. Mele, F. Caldera, F. Trotta and Y. Inoue, Chem. Commun., 2013, 49, 3510.

33 B. V. Slaughter, S. S. Khurshid, O. Z. Fisher, A. Khademhosseini and N. A. Peppas, Adv. Mater., 2009, 21(32-33), 3307.

34 F. Brandl, F. Kastner, R. M. Gschwind, T. Blunk, J. Tessmar and A. Gopferich, J. Controlled Release, 2010, 142, 221.

35 M. Biondi, F. Ungaro, F. Quaglia and P. A. Netti, Adv. Drug Delivery Rev., 2008, 60, 229-242.

36 V. Crupi, D. Majolino, A. Mele, L. Melone, C. Punta, B. Rossi, F. Toraldo, F. Trotta and V. Venuti, Soft Matter, 2014, 10, 2320.

37 V. Crupi, A. Fontana, D. Majolino, A. Mele, L. Melone, C. Punta, B. Rossi, F. Rossi, F. Trotta and V. Venuti, J. Inclusion Phenom. Macrocyclic Chem., 2014, 80, 69.

38 F. Castiglione, V. Crupi, D. Majolino, A. Mele, L. Melone, W. Panzeri, C. Punta, B. Rossi, F. Trotta and V. Venuti, J. Inclusion Phenom. Macrocyclic Chem., 2014, 80, 77.

39 V. Crupi, D. Majolino, A. Mele, B. Rossi, F. Trotta and V. Venuti, Soft Matter, 2013, 9, 6457.

40 F. Castiglione, V. Crupi, D. Majolino, A. Mele, B. Rossi, F. Trotta and V. Venuti, J. Raman Spectrosc., 2013, 44, 1463.

41 V. Venuti, B. Rossi, A. Mele, L. Melone, C. Punta, F. Trotta, V. Crupi, D. Majolino, M. Giarola, G. Mariotto and A. Fontana, 2015, submitted.

42 V. Crupi, A. Fontana, M. Giarola, D. Majolino, G. Mariotto, A. Mele, L. Melone, C. Punta, B. Rossi, F. Trotta and V. Venuti, J. Raman Spectrosc., 2013, 44, 1457.

43 V. Crupi, A. Fontana, M. Giarola, S. Longeville, D. Majolino, G. Mariotto, A. Mele, A. Paciaroni, B. Rossi, F. Trotta and V. Venuti, J. Phys. Chem. B, 2014, 118, 624.
44 A. I. Chumakov, I. Sergueev, U. van Bürck, W. Schirmacher, T. Asthalter, R. Rüffer, O. Leupold and W. Petry, Phys. Rev. Lett., 2004, 92, 245508.

45 T. Deschamps, C. Martinet, D. de Ligny, J. L. Bruneel and B. Champagnon, J. Chem. Phys., 2011, 134, 234503.

46 L. Hong, B. Begen, A. Kisliuk, C. Alba-Simionesco, V. N. Novikov and A. P. Sokolov, Phys. Rev. B: Condens. Matter Mater. Phys., 2008, 78, 134201.

47 M. Ferro, F. Castiglione, C. Punta, L. Melone and W. Panzeri, Beilstein J. Org. Chem., 2014, 10, 2715.

48 G. Guella, I. Mancini, G. Mariotto, B. Rossi and G. Viliani, Phys. Chem. Chem. Phys., 2009, 11, 2420.

49 F. Mallamace, M. Broccio, C. Corsaro, A. Faraone, D. Majolino, V. Venuti, L. Liu, C. Y. Mou and S. H. Chen, Proc. Natl. Acad. Sci. U. S. A., 2007, 104, 424.

50 J. B. Brubach, A. Mermet, A. Filabozzi, A. Gerschel, D. Lairez and M. P. Krafft, J. Phys. Chem. B, 2001, 105, 430.

51 V. Crupi, D. Majolino, F. Longo, P. Migliardo and V. Venuti, Vib. Spectrosc., 2006, 42, 375.

52 G. E. Walrafen, M. S. Hokmababi and W. H. Yang, J. Chem. Phys., 1986, 85, 6964.

53 A. Millo, Y. Raichlin and A. Katzir, Appl. Spectrosc., 2005, 59, 460. 54 B. Rossi, V. Venuti, F. D’Amico, A. Gessini, F. Castiglione, A. Mele, C. Punta, L. Melone, V. Crupi, D. Majolino, F. Trotta and C. Masciovecchio, Phys. Chem. Chem. Phys., 2015, 17, 963.

55 M. M. Coleman, J. F. Graf and P. C. Painter, Specific Interactions and the Miscibility of Polymer Blends, Technomic Publishing Co., Inc., Lancaster, Pennsylvania, 1991.

56 V. Crupi, F. Longo, D. Majolino and V. Venuti, J. Chem. Phys., 2005, 123, 154702.

57 V. Crupi, D. Majolino, P. Migliardo, V. Venuti and

M. C. Bellissent-Funel, Mol. Phys., 2003, 101, 3323.

58 R. Bergman and J. Swanson, Nature, 2000, 403, 283.

59 G. Bellavia, L. Paccou, S. Achir, Y. Guinet, J. Siepmann and

A. Hédoux, Food Biophys., 2013, 8, 170.

60 J. R. Scherer, M. K. Go and S. J. Kint, J. Phys. Chem., 1974, 78, 1304.

61 T. T. Wall and D. F. J. Hornig, J. Chem. Phys., 1965, 43, 2079.

62 W. F. Murphy and H. J. J. Bernstein, J. Phys. Chem., 1972, 76, 1147.

63 A. Hédoux, Y. Guinet and L. J. Paccou, J. Phys. Chem. B, 2011, $115,6740$.

64 G. D’Arrigo, G. Maisano, F. Mallamace, P. Migliardo and F. Wanderlingh, J. Chem. Phys., 1981, 75, 4264.

65 G. E. J. Walrafen, J. Chem. Phys., 1967, 47, 114.

66 P. A. Giguère, J. Chem. Phys., 1987, 87, 4835.

67 D. A. Schmidt and K. Miki, J. Phys. Chem. A, 2007, 111, 10119. 68 F. D’Amico, M. Saito, F. Bencivenga, M. Marsi, A. Gessini, G. Camisasca, E. Principi, R. Cucini, S. Di Fonzo, A. Battistoni, E. Giangrisostomi and C. Masciovecchio, Nucl. Instrum. Methods, 2013, A703, 33.

69 F. Castiglione, V. Crupi, D. Majolino, A. Mele, B. Rossi, F. Trotta and V. Venuti, J. Phys. Chem. B, 2012, 116, 7952.

70 V. Crupi, F. Longo, D. Majolino and V. Venuti, J. Phys.: Condens. Matter, 2006, 18, 3563. 